\title{
Modular Zwitterion-Functionalized Poly(Isopropyl Methacrylate) Polymers for Hosting Luminescent Lead-Halide Perovskite Nanocrystals
}

\author{
Theodore A. Cohen, ${ }^{\dagger, a}$ Yunping Huang, ${ }^{\dagger, b}$ Nico A. Bricker, ${ }^{c}$ Connor S. Juhl, ${ }^{c}$ Tyler J. Milstein, ${ }^{c}$ \\ J. Devin Mackenzie, ${ }^{\text {,d }}$ Christine K. Luscombe, ${ }^{*, a, b, c}$ Daniel R. Gamelin*,a,c \\ ${ }^{a}$ Molecular Engineering and Sciences Institute, University of Washington, Seattle, WA 98195 \\ ${ }^{b}$ Department of Materials Science and Engineering, University of Washington, \\ Seattle, WA 98195 \\ ${ }^{c}$ Department of Chemistry, University of Washington, Seattle, WA 98195-1700 \\ ${ }^{d}$ Department of Mechanical Engineering, University of Washington, Seattle, WA 98195 \\ *Email gamelin@uw.edu; luscombe@uw.edu \\ These authors contributed equally to this work.
}

\begin{abstract}
Inorganic lead-halide perovskite nanocrystals (NCs) are an exciting class of luminescent materials with high defect tolerance and broad spectral tunability, but such NCs are vulnerable to degradation under ambient conditions. Here, we report a class of modular zwitterion-functionalized isopropyl methacrylate polymers designed to stabilize a wide variety of perovskite NCs of different compositions, while also enabling processing in green solvents. Specifically, we report polymers in which the zwitterion spacing is tuned to accommodate the different lattice parameters of $\mathrm{CsPb}\left(\mathrm{Cl}_{1-x} \mathrm{Br}_{x}\right)_{3}$ and $\mathrm{CsPbI}_{3} \mathrm{NCs}$, and we report partially fluorinated polymers prepared to accommodate the needs of infrared-emitting NCs. We show that as-synthesized $\mathrm{CsPbBr}_{3}, \mathrm{CsPbI}_{3}$, and $\mathrm{Yb}^{3+}: \mathrm{CsPbCl}_{3} \mathrm{NCs}$ are easily transferred into these zwitterionic polymers via a simple ligand-exchange procedure. These NC/polymer composites were then cast into thin films that showed substantially improved photoluminescence (PL) and stability compared with more conventional NC/polymer films. Specifically, $\mathrm{CsPbBr}_{3}$ and $\mathrm{CsPbI}_{3}$ NCs in films of their appropriately designed polymers had PL quantum yields of $~ 90 \%$ and $\sim 80 \%$, respectively. PL quantum yields decreased under continuous illumination, but self-healed completely after dark storage. We also found that all the $\mathrm{NC}$ compositions studied here maintain their PL quantum yields in NC/polymer composite films even after 1 year of ambient storage. These encouraging results demonstrate the utility of such modular zwitterion-functionalized polymers for hosting specific perovskite NCs, potentially opening avenues for robust new photonic applications of this important class of NCs.
\end{abstract}




\section{Introduction}

Lead-halide perovskite nanocrystals (NCs) have been heavily investigated for their intriguing properties, ${ }^{1-5}$ including broad spectral tunability with anion ${ }^{6-8}$ and cation ${ }^{9-13}$ replacement, unique electronic structure, ${ }^{14-16}$ and high defect tolerance. ${ }^{17-20}$ These properties have made such NCs a popular emitter for application in light-emitting diodes (LEDs), ${ }^{21-22}$ lasers, ${ }^{23-26}$ single-photon sources, ${ }^{27-29}$ and luminescent solar concentrators. ${ }^{30-33}$ Unfortunately, these NCs are sensitive to degradation in the presence of oxygen and water. ${ }^{34-35}$ To bridge the gap between laboratory research and commercial application, NC stability needs to be improved without sacrificing optoelectronic properties or processability. Furthermore, the broad range of potential applications means that different functional polymer matrices may be required for different uses. For instance, whereas as-synthesized NCs can be supported in various apolar polymers, ${ }^{30,36-40}$ some applications require that NCs are suspended in silicone, ${ }^{21,41-43}$ aqueous, ${ }^{44}$ fluorinated,,${ }^{32,45-47}$ or semiconducting ${ }^{48-50}$ matrices to enable the desired functionalities. Because these host matrices have limited chemical compatibility with the aliphatic ligands often used to stabilize perovskite NCs, additional interface modifiers must be introduced to improve compatibility between the native NC surface ligands and the polymer. Alternatively, these ligands must be replaced with novel ligands tailored to be compatible with the host polymer of interest.

The interface modifier approach has been demonstrated by direct synthesis of perovskite NCs in a hydrophobically stabilized micelle of lauryl methacrylate ${ }^{40}$ and by using custom polymers that form micelles to stabilize the NCs in different hosts, ${ }^{44,51-54}$ with varying levels of success. Novel-ligand approaches have mostly focused on ammonium, ${ }^{21,48-49,55-57}$ carboxylate, ${ }^{58}$ and poly(vinylidene difluoride $)^{22,59} \mathrm{NC}$ coordination. These binding groups have limited affinity for perovskite NC surfaces, ${ }^{60}$ however, and some are consumed during subsequent polymerization 
reactions in making the $\mathrm{NC} /$ polymer composites. ${ }^{61}$ Consequently, most of these polymersuspension protocols yield samples with poor dispersion and reduced NC photoluminescence quantum yields (PLQYs). Alternatively, strongly binding phosphonate, ${ }^{62-66}$ sulfonate, ${ }^{18}$ and Zwitterionic $^{67-68}$ ligands have recently been used to synthesize perovskite NCs with improved PLQYs and stability. The covalently tethered positive and negative ions on zwitterionic ligands are well matched to the highly ionic surface of the NCs. These ligands are mostly aliphatic, however, which limits their ability to solvate NCs in many non-aliphatic functional polymers, complicating device fabrication and ultimately limiting the application range of perovskite NCs. Zwitterionic polymers have been used to passivate perovskite thin films, ${ }^{69-70}$ and while this manuscript was in preparation, zwitterionic polymers were reported to stabilize $\mathrm{CsPbBr}_{3} \mathrm{NCs}_{\text {in }}$ solution and solid state. ${ }^{71-72}$ These works solidify the idea that zwitterionic polymers have high potential as perovskite NC host matrices.

Here, we describe a set of modular zwitterion-functionalized poly(isopropyl methacrylate) polymers developed to match the specific needs of various colloidal lead-halide perovskite NCs. In general, polymers with appended alkyl chains have high solubility in organic solvents, ${ }^{73-75}$ but branched alkyl structures can also improve polymer solubility in green solvents that are sufficiently apolar to prevent $\mathrm{NC}$ degradation ${ }^{76}$ and inhibit the formation of crystalline scattering structures in a solid composite. ${ }^{77-78}$ Consequently, isopropyl methacrylate was used instead of commonly used long-chain alkyl acrylates; the increased solubility from the isopropyl side chain facilitates solution processing with green solvents such as butyl acetate that are still sufficiently apolar to prevent NC degradation. Within these polymers, the distances between positive quaternary ammonium moieties and negative sulfonate moieties are tuned using either 3- or 4methylene spacers. This tunability allows the zwitterion separation to be matched with the 
relevant $\mathrm{NC}$ lattice spacings when the $\mathrm{NC}$ anions are changed from $\mathrm{Cl}^{-} / \mathrm{Br}^{-}$to $\mathrm{I}^{-}{ }^{68}$ We show that the native NC ligands are easily replaced by these zwitterionic polymers, and the resulting polymers are shown to stabilize lead-halide perovskite NCs across the entire composition and luminescence-color range. For $\mathrm{Yb}^{3+}$-doped NCs with near-infrared emission, we further utilize a fluorinated version of poly(isopropyl methacrylate) that reduces absorption from $\mathrm{C}-\mathrm{H}$ vibrational overtones at $\mathrm{Yb}^{3+}$ emission at infrared wavelengths. ${ }^{32,79}$ The modularity of these polymers provides access to a variety of perovskite NC/polymer composites with attractive solution processability, optical properties, and long-term stability, making these polymers appealing hosts for use of colloidal lead-halide perovskite NCs in various photonic applications.

\section{Results and Analysis}

Preparation of zwitterion-functionalized polymers. Scheme 1 summarizes the approach used to synthesize modular zwitterion-functionalized polymers. We used 2,2'-azobis(2methylpropionitrile) (AIBN) to initiate the radical copolymerization of two commercial acrylate monomers, 2-(dimethylamino)ethyl acrylate and isopropyl methacrylate (or fluorinated isopropyl methacrylate) in a 1:9 ratio, to obtain amine-precursor polymers. This approach allows adaptation for many other functional groups simply by replacing isopropyl methacrylate with other acrylate or methacrylate monomers. Thiol, a chain transfer agent, was added to the polymerization reaction to prevent formation of high-molecular-weight polymers with reduced solubility. These amine-precursor polymers were then reacted with the corresponding sultones to yield the desired zwitterion-functionalized polymers. This flexible chemistry allows integration of zwitterions with different anion-cation separations (3 or 4 methylene spacers). For this work, we successfully synthesized 3-carbon-separated zwitterion-functionalized polymers (ZP3) and their fluorinated analogs (ZFP3), as well as 4-carbon-separated zwitterion-functionalized 
polymers (ZP4) and their fluorinated analogs (ZFP4). ${ }^{1} \mathrm{H}$-nuclear magnetic resonance (NMR) spectra of all four polymers are provided in the Supporting Information.

Scheme 1. Summary of the synthesis of a series of zwitterion-functionalized polymers. The ratio $1: \mathrm{m}$ is $1: 9$ for all polymers reported here.

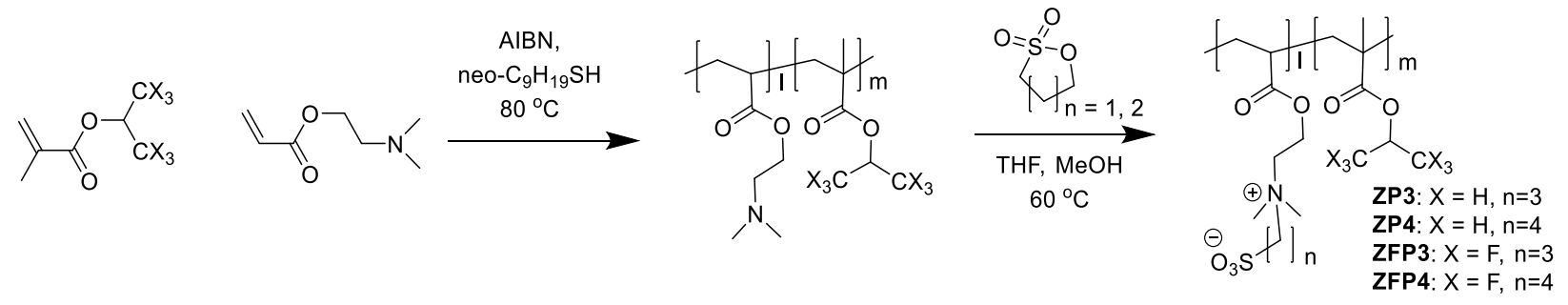

Three of these polymers were then used for hosting three different categories of perovskite NCs, as summarized in Table 1: undoped NCs with small lattice parameters (e.g., $\mathrm{CsPbBr}_{3} \mathrm{NCs}$ ), large lattice parameters (e.g., $\mathrm{CsPbI}_{3} \mathrm{NCs}$ ), and doped $\mathrm{NCs}$ showing NIR emission (e.g., $\left.\mathrm{Yb}^{3+}: \mathrm{CsPbCl}_{3} \mathrm{NCs}\right)$.

Table 1. Summary of the zwitterionic polymers developed in this work and the perovskite NC/polymer composites prepared from each. The fluorinated polymer with 4carbon zwitterion spacing was successfully synthesized but was not soluble in ethyl acetate.

\section{Zwitterion}

Abbreviation

ZP3

$\mathrm{ZP} 4$

ZFP3

separation

3 carbons

4 carbons

3 carbons
Fluorinated

No

No

Yes NCs used $\mathrm{CsPbBr}_{3}$

$\mathrm{CsPbI}_{3}, \mathrm{CsPb}\left(\mathrm{Br}_{1-x} \mathrm{I}_{x}\right)_{3}$ $\mathrm{CsPbBr}_{3}, \mathrm{Yb}^{3+}: \mathrm{CsPbCl}_{3}$
Amt. ZP used per 3 pico-mol NC

$77 \mathrm{mg}$

$7 \mathrm{mg}$

$120 \mathrm{mg}$

CsPbBr3 NC/polymer composites. Figure 1a shows ${ }^{1} \mathrm{H}-\mathrm{NMR}$ spectra of ZP3 before and after the $\mathrm{CsPbBr}_{3} \mathrm{NC}$ ligand exchange from native ligands to zwitterionic polymers. There is no signal at 5.5-6.0 ppm attributing to the $\mathrm{C}=\mathrm{C}$ bond of $\mathrm{OA}$ or $\mathrm{OAm}$ in the pre-ligand-exchanged sample. ${ }^{6}$ This result indicates that the original NC ligands are fully removed in the ligand exchange. In the ligand-exchanged sample, we do notice a singlet centered at $0.12 \mathrm{ppm}$, which is 
assigned to TMS-acetate, ${ }^{80}$ indicating incomplete removal of acetate groups during $\mathrm{NC}$ preparation. No signals from TMS-Br $(0.36 \mathrm{ppm})$ or TMS-sulfonate $(0.40 \mathrm{ppm}$, the product after TMS-Br reacts with sulfonate groups) were detected. This result indicates that the TMS-halide precursors were all consumed during synthesis and converted to TMS-acetate, a molecule that is likely a spectator in subsequent procedures. NMR signals associated with the polymer backbone appear between 0.7 and $2.5 \mathrm{ppm}$. The additional peaks between 2.7 and $5.2 \mathrm{ppm}$ are assigned according to the color-coded diagram in the inset of Figure 1a. Most NMR peaks remain essentially unchanged upon NC addition, but the reduced electron density of the sulfonate anion upon association with NC surface cations leads to a shift of the adjacent methylene protons from 2.93 to $3.05 \mathrm{ppm}$ (highlighted magenta). ${ }^{81-85}$ For the quaternary ammonium ion, the NMR data show that only one methylene group is closely associated with the NC surfaces (highlighted green), while the other methyl/methylenes are not. The NMR signal of the $\mathrm{H}$ atoms on this closely interacting methylene group have the highest frequency of all the methyl/methylene groups on the quaternary ammonium ion. This result indicates that those $\mathrm{H}$ atoms have the lowest electron density and thus are more likely to interact with halide anions of the NC surfaces. These NC polymer composites were soluble in butyl acetate, a green solvent that is a common antisolvent for perovskite NCs. ${ }^{86}$ Furthermore, the solution-state NC PLQY in a given zwitterionic polymer did not increase as the NC concentration was increased (see Supporting Information), indicating that the zwitterionic polymers are strongly bound to the NCs. ${ }^{18,65}$ The NCs in a zwitterionic polymer solution had a maximum PLQY of $84 \pm 5 \%$ compared with $59 \pm$ $5 \%$ for the same as-synthesized NCs, indicating that surface defects are effectively passivated by the zwitterions on these polymers. 

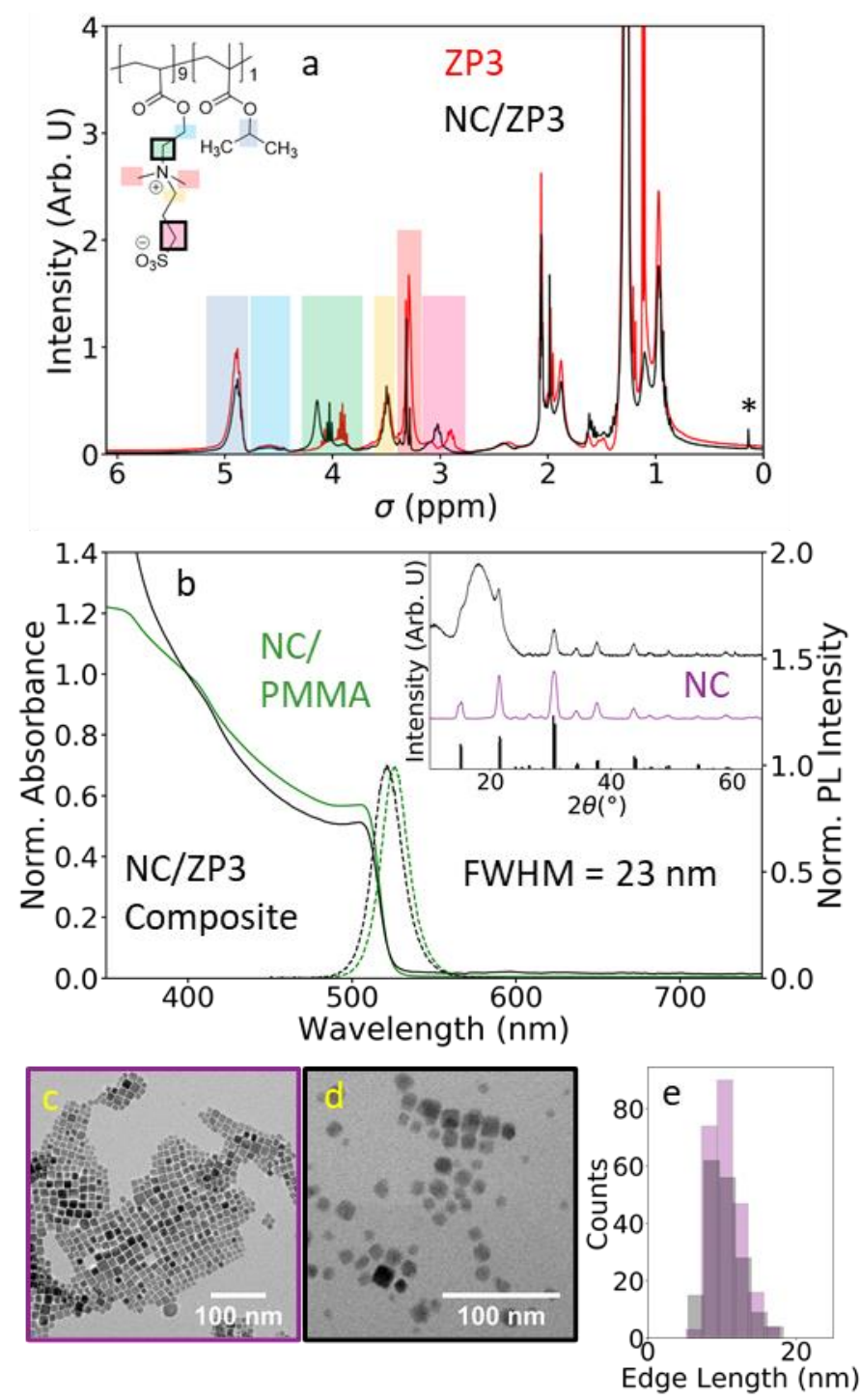

Figure 1. (a) ${ }^{1} \mathrm{H}-\mathrm{NMR}$ spectra of $\mathrm{ZP} 3$ in acetone- $\mathrm{d}_{6}$ before and after binding to $\mathrm{CsPbBr}_{3}$ NCs. The peaks around 3 and 4 ppm are substantially shifted after NC binding, indicating that the protons associated with these peaks (bolded boxes) are confined near NC surfaces. * indicates the peak associated with TMS-acetate. (b) Absorption and PL spectra of $\mathrm{CsPbBr}_{3} \mathrm{NC}$ /polymer composites drop cast from a solution of $\mathrm{NCs}$ and $\mathrm{ZP} 3$ in butyl acetate and a solution of NCs and PMMA in toluene. Absorption spectra are normalized at $400 \mathrm{~nm}$ and PL spectra are normalized to the PL maximum. The PMMA sample was cast in a $\mathrm{N}_{2}$-filled glovebox to maximize PLQY. Inset: XRD data collected before and after transfer of NCs to ZP3. The broad peaks from $\sim 10$ to $\sim 17^{\circ}$ are attributed to scattering by the amorphous polymer. TEM images of $\mathrm{CsPbBr}_{3} \mathrm{NCs}$ (c) drop cast from hexanes solution and (d) drop cast from a solution of NC/ZP3 composite in butyl acetate. (e) Histogram of edge lengths from the TEM images shown in panel b and Figure $S 9$.

Once purified, the $\mathrm{CsPbBr}_{3} \mathrm{NC} / \mathrm{ZP} 3$ solution was drop cast onto clean glass slides to form composite thin films. Figure $1 \mathrm{~b}$ shows absorption and photoluminescence (PL) spectra of 
$\mathrm{CsPbBr}_{3} \mathrm{NCs}$ in a $\mathrm{ZP} 3$ film and, for comparison, the same NCs in a high-molecular-weight poly(methyl methacrylate) (PMMA) composite (see Supporting Information). Apart from small reabsorption- and aggregation-induced shifts in the PL (see Supporting Information), the UV/vis and PL spectra are nearly unchanged after the NCs are transferred into a ZP3 composite and the full width at half-maximum (FWHM) of the NC PL is sufficiently small for applications in LEDs with precise green color rendering. ${ }^{87}$ The NC/ZP3 composite has no detectable sub-bandgap scattering, indicating that it has few NC aggregates. ${ }^{88}$

The inset of Figure 1b shows X-ray diffraction (XRD) data collected from as-synthesized $\mathrm{CsPbBr}_{3} \mathrm{NCs}$ and the same NCs in a $\mathrm{ZP3}$ composite. Apart from additional amorphous scattering signals, the diffraction peaks are the same for both samples. Because each peak in the XRD consists of multiple Bragg reflections derived from the orthorhombic $\mathrm{CsPbBr}_{3}$ crystal structure, we could not perform a precise Scherrer analysis. That said, although the XRD FWHM for the NC diffraction peak at $44^{\circ}$ increases by $\sim 0.006^{\circ}(0.7 \%)$ in the $\mathrm{ZP} 3$ composite, this difference is likely within experimental uncertainty. Furthermore, the transmission electron microscope (TEM) images in Figure 1c,d show that the $\mathrm{CsPbBr}_{3} \mathrm{NCs}$ maintain their cube-like shapes in a ZP3 composite. The dark spots in both images are attributed to $\mathrm{Pb}^{0}$ nanoparticles formed during TEM imaging. Figure 1e plots histograms of nanocrystal edge lengths taken from the images in Figure 1c,d and Figure S9. The average NC edge lengths before and after binding by ZP3 are $10.6 \pm 2.1 \mathrm{~nm}$ and $10.4 \pm 2.4 \mathrm{~nm}$, respectively. Importantly, the solid-state PLQYs of the $\mathrm{CsPbBr}_{3} \mathrm{NCs}$ in PMMA and ZP3 composites were measured to be $55 \pm 5 \%$ and $90 \pm 5 \%$, respectively. These PLQYs suggest that non-radiative recombination sites are effectively passivated when the NCs are transferred into ZP3.

The stabilities of the NC composites were assessed in ambient atmosphere both in the dark 
and under illumination. In initial experiments, we note that $\mathrm{CsPbBr}_{3} \mathrm{NC} /$ zwitterionic polymer solutions in butyl acetate stored in ambient conditions maintained $~ 95 \%$ of their initial PLQY after 5 months of dark storage (see Supporting Information), whereas as-synthesized NCs in hexanes generally precipitate from solution after several days of ambient dark storage. To understand how these observations might translate to solid-state samples, we performed systematic stability measurements on various solid composites. To probe the PL stability of these NC composites, Figure 2a plots PLQYs of solid $\mathrm{CsPbBr}_{3} \mathrm{NC} / \mathrm{ZP} 3$ and NC/PMMA composites measured as a function of ambient dark storage time. The NCs showed similar long-term dark storage stability in ZP3 as in PMMA, but the PLQY of the NC/ZP3 composite was 2 times greater, indicating the efficacy of these zwitterionic polymers as a ligating matrix. XRD data (Supporting Information, Figure S11) show that $\mathrm{CsPbBr}_{3} \mathrm{NCs}$ in solid zwitterionic polymer composites maintain their crystal structure after 9 months of dark storage and PLQY data show that the PL is preserved after 1 year of dark storage. Both results are indications of the resilience of these NCs in zwitterionic polymer composites.

Figure $2 b$ plots PLQYs of various NC/polymer composites measured as a function of illumination time under ambient atmosphere, using $\sim 90 \mathrm{~mW} \mathrm{~cm} \mathrm{~cm}^{-2}$ of full-area, $450 \mathrm{~nm}$ irradiation. The NC/ZP3 sample loses about $20 \%$ of its absolute PLQY in the first 12 hrs of illumination, but the NC PL intensity remains constant through the remaining $75 \mathrm{hrs}$ of the experiment. Furthermore, the PLQYs of the NC/ZP3 samples recover over multiple time scales. For example, Figure S13 shows an initial PLQY loss $(5-10 \mathrm{sec})$ that fully recovers after $\sim 10 \mathrm{~min}$ of dark storage, and Figure 2b shows that both the rapid PLQY loss and the slower PLQY decrease $(\sim 12 \mathrm{hrs})$ recover to $\sim 90 \%$ of the initial PLQY after 6 days of dark storage. In contrast, the NC/PMMA sample loses almost all of its PLQY after 24 hrs of irradiation, and this PLQY 
does not recover with dark storage. This result is consistent with the observation that perovskite NC/PMMA composites cannot withstand the high fluences $\left(>100 \mathrm{~W} \mathrm{~cm}^{-2}\right)$ of single-particle spectroscopic measurements in ambient conditions. ${ }^{89}$ Most reports of related NC/polymer composites do not perform irradiation stability measurements on the timescale needed to observe the rapid PLQY loss observed in Figure S13, so it is unclear whether this drop is even more general among perovskite NCs.
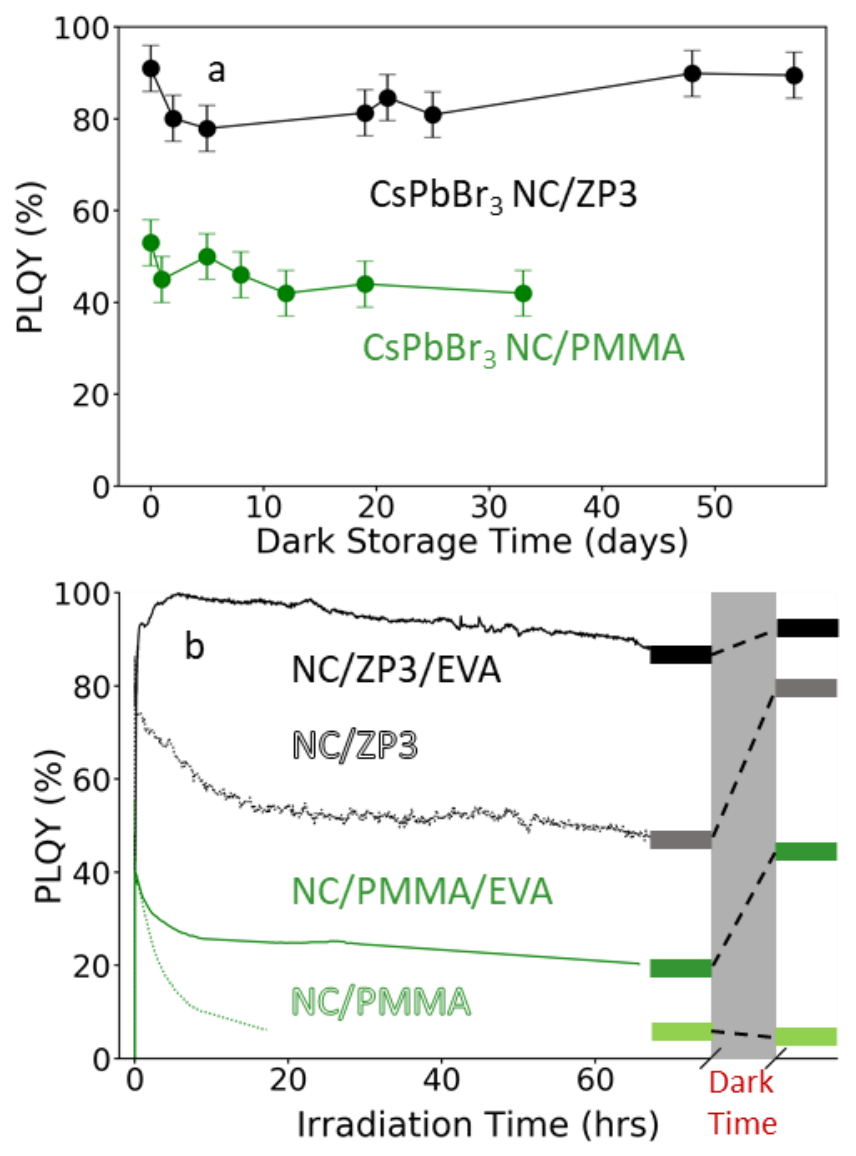

Figure 2. (a) PLQYs as a function of dark storage time under ambient atmosphere for drop-cast $\mathrm{CsPbBr}_{3} \mathrm{NCs}$ in PMMA and ZP3 composites. The NCs in ZP3 preserve all their original PLQY over 2 months while NCs in PMMA lose 20\% of their PLQY over the first 15 days of dark storage. (b) PLQYs plotted as a function of $450 \mathrm{~nm}$ irradiation time for $\mathrm{CsPbBr}_{3} \mathrm{NC/ZP3}$ and NC/PMMA composites with and without EVA encapsulation. The PLQYs were measured again after each sample was stored in the dark for several days following the irradiation experiment, and the values measured before and after dark storage are indicated as horizontal bars. 
To better evaluate the potential of these NC/polymer composite in commercial applications, we laminated $\mathrm{CsPbr}_{3} \mathrm{NC} / \mathrm{ZP} 3$ and NC/PMMA samples between two layers of glass with poly(ethylene-co-vinyl acetate) (EVA) to reduce exposure of the NCs to air. Remarkably, the PLQY of the EVA-laminated NC/ZP3 sample increases to nearly $100 \%$ within the first few hours of illumination and stays constant at this value for the remaining $75 \mathrm{hrs}$ of the experiment. Ex situ integrating-sphere measurements verify that after $75 \mathrm{hrs}$ of irradiation, the PLQY of the EVA-laminated sample increased from $48 \pm 5 \%$ to $93 \pm 5 \%$; this PLQY is among the highest reported to date for any inorganic or hybrid bromo-perovskite NC/polymer solids. ${ }^{22,51,58,90} \mathrm{We}$ also note that the PL spectra of both samples do not change substantially over the course of this measurement (see Supporting Information), suggesting that this photo-brightening is not attributable to irreversible etching of the NCs. ${ }^{27,37}$ In contrast, the PLQY of the EVA-laminated NC/PMMA sample decreased under illumination, recovering again after dark storage. These irradiation results indicate that the photostability of $\mathrm{CsPbBr}_{3} \mathrm{NCs}$ can be substantially enhanced by limiting air exposure. Additionally, when these NCs are hosted in a ZP3 composite, the high density of zwitterionic passivating groups in this polymer improves photostability in ambient conditions and increases PLQY in inert conditions relative to the same NCs in PMMA.

$\operatorname{CsPb}\left(\operatorname{Br} 1_{-x} I_{x}\right)_{3} \mathbf{N C / p o l y m e r ~ c o m p o s i t e s . ~ W e ~ n o w ~ t u r n ~ o u r ~ a t t e n t i o n ~ t o ~ p o l y m e r ~ c o m p o s i t e s ~}$ that stabilize red-emitting $\mathrm{CsPbI}_{3} \mathrm{NCs}$. The $\mathrm{CsPbI}_{3} \mathrm{NCs}$ were found to be noticeably less stable than $\mathrm{CsPbr}_{3} \mathrm{NCs}$ upon mixing with the zwitterionic polymers. For example, to prevent $\mathrm{NC}$ dissolution during mixing, it was necessary to use $\sim 10 \mathrm{x}$ less polymer per of NC. Even when $\mathrm{NC}$ /polymer solutions were deposited onto substrates immediately after ligand exchange, XRD data (see Supporting Information) show a larger increase in the FWHM of the $28^{\circ}$ peak of the $\gamma$ - 
cubic phase $\mathrm{CsPbI}_{3} \mathrm{NCs}$ in $\mathrm{ZP} 4$ than was noted for $\mathrm{CsPbBr}_{3} \mathrm{NCs}$ in $\mathrm{ZP}$, suggesting that the $\mathrm{CsPbI}_{3} \mathrm{NCs}$ are etched during polymer binding. Additionally, although $\mathrm{CsPbBr}_{3} \mathrm{NC} / \mathrm{ZP} 3$ composites in butyl acetate were stable under ambient conditions for up to 5 months, $\mathrm{CsPbI}_{3}$ NC/ZP4 composites in butyl acetate were only stable for $\sim 24$ hrs in similar storage conditions.

Fortunately, the $\mathrm{CsPbI}_{3} \mathrm{NCs}$ in ZP4 show significantly improved PL compared to the same NCs either drop cast without polymer or embedded in PMMA composites. Figure 3a plots absorption and PL spectra of a $\mathrm{CsPbI}_{3} \mathrm{NC}_{\mathrm{ZP}} 4$ composite and, for comparison, also of a $\mathrm{CsPbI}_{3}$ NC/PMMA composite. The absorption and PL spectra for NCs in ZP4 and PMMA are nearly identical, apart from greater sub-bandgap scattering and a slight PL blue shift in the NC/ZP4 sample. The PL decay of the NCs in ZP4 is nearly monoexponential, whereas that of the NCs in PMMA appears biexponential (see Supporting Information). The PLQYs of the NCs in ZP4 and PMMA composites are $85 \pm 5 \%$ and $45 \pm 5 \%$, respectively, suggesting that the additional PL decay component of the NCs in a PMMA composite can be attributed to non-radiative recombination at non-passivated surface traps. We also successfully stabilized $\mathrm{CsPb}\left(\mathrm{Br}_{1-x} \mathrm{I}_{x}\right)_{3}$ NCs in ZP4 composites, but observed a $50 \mathrm{~nm}$ PL blue shift after polymer binding. The PLQY of $\mathrm{CsPb}\left(\mathrm{Br}_{1-x} \mathrm{I}_{x}\right)_{3} \mathrm{NC} / \mathrm{ZP} 4$ composite was only $\sim 30 \%$ (see Supporting Information). 

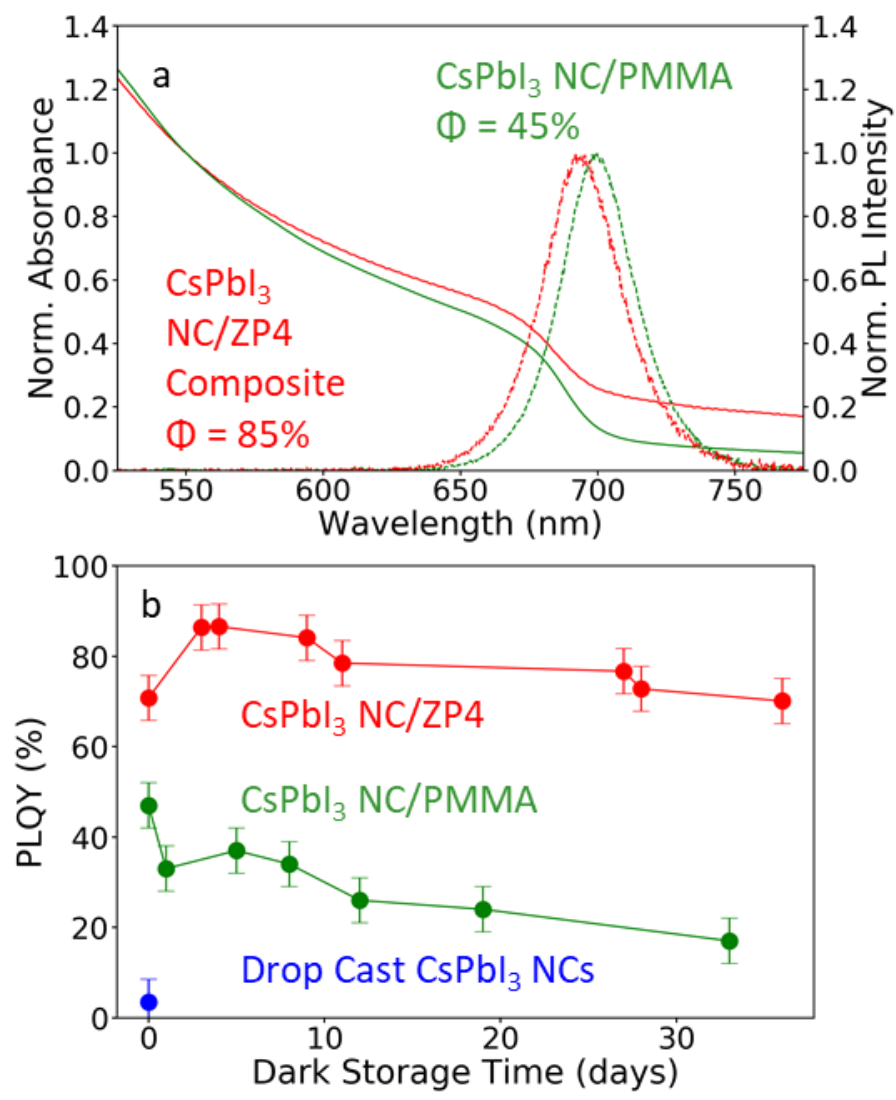

Figure 3. (a) Absorption and PL spectra of $\mathrm{CsPbI}_{3} \mathrm{NCs}$ in $\mathrm{ZP} 4$ and PMMA composites. Absorption spectra are normalized at $550 \mathrm{~nm}$ and PL spectra are normalized to the PL maximum. The PLQYs of these samples are also indicated. (b) $\mathrm{CsPbI}_{3} \mathrm{NC}$ PLQYs plotted as a function of dark storage time in ambient atmosphere, measured for $\mathrm{CsPbI}_{3}$ NC/ZP4 and NC/PMMA composites and the same NCs drop cast from hexane solution.

Figure $3 b$ plots PLQYs of $\mathrm{CsPbI}_{3}$ NCs drop cast onto a glass substrate along with $\mathrm{CsPbI}_{3}$ $\mathrm{NC} / \mathrm{ZP} 4$ and $\mathrm{CsPbI}_{3} \mathrm{NC} / \mathrm{PMMA}$ composites, measured as a function of dark storage time under ambient atmosphere. The drop-cast $\mathrm{CsPbI}_{3} \mathrm{NCs}$ degraded almost as soon as they were exposed to air, and the NCs in PMMA lost two thirds of their PLQY over the course of $~ 32$ days, dropping to $18 \pm 5 \%$. In contrast, the NCs in ZP4 maintain their PLQY well over the entire 36day experiment, indicating improved long-term stability of $\mathrm{CsPbI}_{3} \mathrm{NCs}$ embedded within the ZP4 polymer matrix. Additionally, XRD data (Supporting Information, Figure S14) show that the same $\mathrm{CsPbI}_{3} \mathrm{NC}$ crystal structure is maintained after 9 months of dark storage and the PLQY is maintained after 1 year of dark storage in ambient conditions. We also found that the PLQYs 
of NC/ZP4 and NC/PMMA composites decrease under $450 \mathrm{~nm}$, full area irradiation, but the NC/PMMA composite fully degrades within the first 5 hrs of irradiation, while the NC/ZP4 composite retains some luminescence through the full $100 \mathrm{hrs}$ of irradiation (see Supporting Information). Furthermore, NC/ZP4 composites recover most of their PLQY after dark storage time, while the PLQY of the NC/PMMA composites remain low after dark storage time. These observations demonstrate that, like the $\mathrm{CsPbBr}_{3} \mathrm{NC} / \mathrm{ZP} 3$ composites, $\mathrm{CsPbI}_{3} \mathrm{NCs}$ in $\mathrm{ZP} 4$ show reversible PL degradation when irradiated in the presence of air whereas the same NCs in PMMA show irreversible PL degradation.

$\mathrm{Yb}^{3+}$-doped $\mathrm{CsPbCl}_{3} \mathrm{NC}$ /polymer composites. Figure 4a shows the absorption spectrum of $\mathrm{Yb}^{3+}: \mathrm{CsPbCl}_{3} \mathrm{NCs}$ in a $\mathrm{ZFP} 3$ composite thin film. The corresponding fluorinated polymer without zwitterions is insoluble in the apolar solvents used to process as-synthesized NCs. When zwitterionic groups are added, however, the zwitterionic fluorinated polymer is soluble in butyl acetate and can be used to stabilize as-synthesized $\mathrm{Yb}^{3+}: \mathrm{CsPCl}_{3} \mathrm{NCs}$ via the ligand exchange procedure described here. The absorption spectrum of the NC/ZFP3 film shows minimal subbandgap scattering, indicating that high concentrations of NCs can be well dispersed in ZFP3. The inset to Figure 4a shows a TEM image of the $\mathrm{Yb}^{3+}: \mathrm{CsPbCl}_{3} \mathrm{NCs}$ in ZFP3. This image shows that the $\mathrm{NC}$ structure is similar to those reported previously for colloidal $\mathrm{Yb}^{3+}: \mathrm{CsPbCl}_{3} \mathrm{NCs}^{9}$ Figure $4 \mathrm{~b}$ plots PL spectra of zwitterionic ligand-capped $\mathrm{Yb}^{3+}: \mathrm{CsPCl}_{3} \mathrm{NCs}$ drop cast from solution and of oleylamine-capped NCs after incorporation into a ZFP3 composite, measured such that relative intensities can be compared quantitatively. The PLQY of the NC/ZFP3 film is slightly greater than that of drop-cast NCs, owing to effective surface passivation by zwitterions in ZFP3. Additionally, the NC/ZFP3 film retains nearly the same PLQY for over 1.8 years of dark storage, an indication that the $\mathrm{Yb}^{3+}: \mathrm{CsPCl}_{3} \mathrm{NCs}$ are highly stable in this polymer. 

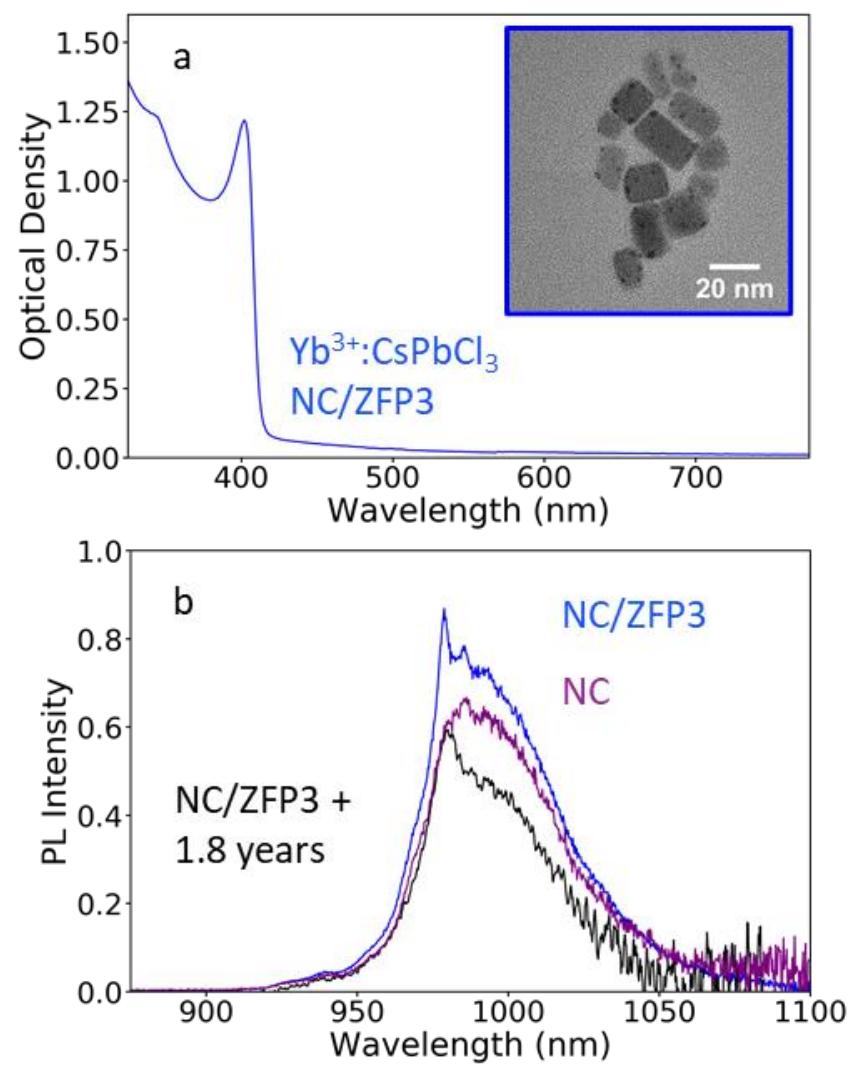

Figure 4. (a) Absorption spectra of a $\mathrm{Yb}^{3+}: \mathrm{CsPbCl}_{3} \mathrm{NC} / \mathrm{ZPF} 3$ composite film drop cast from butyl acetate. Inset: TEM image of these NCs in the NC/ZFP3 composite. (b) NIR $\mathrm{PL}$ spectra of the $\mathrm{Yb}^{3+}: \mathrm{CsPbCl}_{3} \mathrm{NC} /$ polymer composite shown in panel (a). The PL spectra of the NC/ZFP3 composite after 1.8 years of dark, ambient storage and of the drop-cast zwitterionic-ligand capped NCs without polymer are also provided for reference. These PL spectra were measured quantitatively such that relative integrated intensities are proportional to relative PLQYs.

\section{Discussion}

We have developed a series of modular zwitterion-functionalized poly(isopropyl methacrylate) polymers with tailored inter-ion spacings and $\mathrm{C}-\mathrm{H}$ bond densities, and have demonstrated the use of these polymers to host highly luminescent $\mathrm{CsPbBr}_{3}, \mathrm{CsPbI}_{3}$, and $\mathrm{Yb}^{3+}: \mathrm{CsPCl}_{3} \quad \mathrm{NCs}$ in green organic solvents without sacrificing their attractive PL characteristics. For all the NCs investigated here, the PLQYs increased upon incorporation into the zwitterion-functionalized polymers, an observation attributed to effective NC surface 
passivation by the zwitterionic functional groups. These NC/polymer composites could be cast into stable thin films, forming high-optical-quality solids with well-dispersed NCs in most cases. The NCs in these polymers show dark recovery of their PLQYs following extended irradiation in ambient atmosphere, likely aided by the high local concentrations of unbound zwitterion groups in these polymers, which may help to passivate surface defects formed through continuous NC irradiation.

Although many studies have addressed the stability of $\mathrm{CsPBrr}_{3} \mathrm{NCs}$ in polymer matrices, ${ }^{21-}$ 22, 36, 40, 49,51,55, 58, 67, 71-72 $\mathrm{CsPbI}_{3} \mathrm{NCs}$ have proven especially challenging to stabilize in polymers. ${ }^{27,34,91} \mathrm{CsPbI}_{3} \mathrm{NCs}$ have been stabilized in solution with near-unity PLQYs, ${ }^{18,62,92}$ but only one recent report claims to have stabilized $\mathrm{CsPbI}_{3} \mathrm{NCs}$ in a polymer with $\sim 99 \%$ PLQY, obtaining this high PLQY with phosphine additives. ${ }^{66}$ Here, we have demonstrated the use of zwitterion-functionalized polymers to stabilize $\mathrm{CsPbI}_{3} \mathrm{NCs}_{\text {sith }} \sim 80 \%$ PLQY. The polymer was tailored to accommodate the lattice parameters of $\mathrm{CsPbI}_{3} \mathrm{NCs}^{68}$ using zwitterionic functional groups having 4-methylene spacers between sulfonate and quaternary ammonium ions. Furthermore, because $\mathrm{CsPbI}_{3} \mathrm{NCs}$ are less stable against dissolution in polar solvents than their bromide analogues, the branched isopropyl groups of the zwitterion-functionalized polymers introduced here play an important role by enhancing polymer solubility in aprotic solvents. This enables formation of $\mathrm{NC}$ /polymer composites in most organic solvents, including green solvents like butyl acetate that prevent $\mathrm{CsPbI}_{3} \mathrm{NC}$ dissolution over a modest, but acceptable processing window.

In addition to these attributes, the chemistry of the polymers developed here also makes them attractive for hosting perovskite NCs in various contexts. The synthetic strategy described in Scheme 1 is sufficiently modular that both the NC-coordinating and functional groups are 
interchangeable with little modification of reaction procedures. Proof-of-concept results have been demonstrated by swapping the isopropyl methacrylate with fluorinated isopropyl methacrylate. The high NIR transparencies of resultant fluorinated polymers ${ }^{93-94}$ combined with the high solubility and stability of $\mathrm{Yb}^{3+}: \mathrm{CsPCl}_{3} \mathrm{NCs}$ in this fluorinated polymer offers an attractive solution to the problem of rapidly attenuated $\mathrm{Yb}^{3+} \mathrm{NIR}$ emission in optical waveguides made from $\mathrm{C}-\mathrm{H}-$ bond rich polymers that suffer from intense infrared absorption, for example in quantum-cutting luminescent solar concentrators or optical cavities. ${ }^{32}$ In addition to fluorinated isopropyl methacrylate, the modularity of this polymer synthesis suggests that a wide variety of acrylate/methacrylate monomers with various functional groups can be included to modify the physical and chemical properties of these NC composites. For example, poly(ethylene glycol) offers water solubility ${ }^{95}$ and 1-bromo-2,3-epoxypropane allows for crosslinking. ${ }^{96}$ Similarly, although proof-of-concept results have been presented demonstrating stable red-emitting $\mathrm{CsPb}\left(\mathrm{Br}_{1-x} \mathrm{I}_{x}\right)_{3} \mathrm{NC} /$ polymer composite thin films relevant to LEDs, ${ }^{87}$ prior work has suggested that phosphonate-based zwitterionic groups may better stabilize such anion-alloyed perovskite $\mathrm{NCs},{ }^{68}$ and the synthesis methods described here are also amenable to swapping these functional groups. Moreover, because the NC/polymer composites are highly soluble in different organic solvents/mediums, they should also be easily blended and crosslinked with commercial resins without impacting their ability to stabilize perovskite NCs. Such an approach could be used to prepare fully crosslinked NC/polymer composites with various mechanical properties (e.g., flexibility and stretchability) to further expand the functionalities of these NC/polymer composite for most commercial or experimental applications. This class of polymers thus offers broadly attractive chemical flexibility. 


\section{Conclusion}

In summary, we demonstrate a straightforward method to synthesize a full series of novel, modular zwitterion-functionalized poly(isopropyl methacrylate) polymers that can be specifically tailored to host different types of luminescent perovskite NCs. These polymers use tunable zwitterionic anchor groups to stabilize the perovskite NCs, and they use fluorinated or nonfluorinated moieties to tune other optical characteristics, making them useful for hosting a broad array of doped and undoped perovskite NCs. As-prepared perovskite NCs could be easily transferred into these polymers via simple ligand exchange and precipitation, and the resulting perovskite NC/polymer composites show good NC solubility in most solvents, including green solvents like ethyl and butyl acetate. These solvated composites could be easily cast into highoptical-quality solid thin films for further optical interrogation or application. The NC PLQYs and ambient stabilities in these polymers are substantially enhanced relative to those in more conventional PMMA polymer matrices. Overall, these findings establish this class of zwitterionfunctionalized polymers as a flexible platform for advancing fundamental research involving perovskite $\mathrm{NC} /$ polymer composites and may help to advance the commercialization potential of perovskite NCs by facilitating full-scale manufacturing of luminescent NC-based composites via solution processing with green solvents.

\section{Experimental Procedures}

Materials. Lead acetate trihydrate $\left[\mathrm{Pb}(\mathrm{OAc})_{2} \cdot 3 \mathrm{H}_{2} \mathrm{O}\right]$ (99.9\%, Baker Chemical), cesium carbonate $\left[\mathrm{Cs}_{2} \mathrm{CO}_{3}\right]$ (99.9\%, Sigma Aldrich), cesium acetate [CsOAc] (99.9\%, Alfa Aesar), ytterbium acetate hydrate $\left[\mathrm{Yb}(\mathrm{OAc})_{3} \cdot \mathrm{xH}_{2} \mathrm{O}\right](99.9 \%$ Strem Chemical), anhydrous ethanol (200 proof, Decon Laboratories), chlorotrimethylsilane [TMS-Cl] (98\%, Acros Organic), bromotrimethylsilane [TMS-Br] (97\%, Sigma Aldirch), iodotrimethylsilane [TMS-I] (97\%, Sigma Aldirch), 1-octadecene [ODE] (90\%, Sigma Aldrich), oleylamine [OAm] (70\%, Sigma Aldrich), oleic acid [OA] (90\%, Sigma Aldrich), 3-(N,N-Dimethyloctadecylammonio)propanesulfonate [ZW-lig] ( $\geq 99.0 \%$ TLC, Sigma Aldrich), hexanes (99\%, mixture of isomers, Sigma Aldrich), toluene (HPLC, Fischer Chemical), anhydrous ethyl acetate (99\%, Sigma Aldrich), anhydrous butyl acetate (99\%, Sigma Aldrich), anhydrous tetrahydrofuran [THF] $\left(\right.$ Optima $^{\circledR}$, Fischer Chemical), methanol (ACS, EMD Millipore), acetone (ACS, Fischer 
Chemical), 2-propanol (ACS, Fischer Chemical), methanol-d 4 (>99.8 atom \% D, Sigma Aldrich), acetone-d $_{6}$ (99.9 atom \% D, Sigma Aldrich), poly(methyl methacrylate) [PMMA] ( 120,000 MW Sigma Aldrich), tert-Nonyl mercaptan (>97.0\%, mixture of isomers, TCI), 2,2'-azobis(2methylpropionitrile) (98\%, Sigma Aldrich) 2-(dimethylamino)ethyl acrylate $(>98.0 \%$, stabilized with MEHQ, TCI), isopropyl methacrylate (>98.0\%, stabilized with MEHQ, TCI), 1,1,1,3,3,3hexafluoroisopropyl methacrylate (99\%, stab., Alfa Aesar), 1,4-butanesultone (99\%, TCI), 1,3propanesultone (99\%, TCI), Poly(ethylene-co-vinyl acetate) [EVA] (MSL Solar Company), and Spectra/Por ${ }^{\circledR} 6$ dialysis tubing (MWCO 1000) were used as received unless otherwise noted.

Polymer syntheses and purification. Amine precursor polymer syntheses. The polymer synthesis process is outlined in Scheme 1. 2-(Dimethylamino)ethyl acrylate (143 mg, $1 \mathrm{mmol}$ ) and either isopropyl methacrylate (1152 $\mathrm{mg}, 9 \mathrm{mmol})$ or 1,1,1,3,3,3-hexafluoroisopropyl methacrylate $(2124 \mathrm{mg}, 9 \mathrm{mmol})$ was added to a vial that was pre-dried overnight in a $120{ }^{\circ} \mathrm{C}$ oven. 2,2'-azobis(2-methylpropionitrile) $(8 \mathrm{mg}, 0.5 \mathrm{mmol})$ and tert-nonyl mercaptan $(8 \mu \mathrm{L}, 0.5$ $\mathrm{mmol}$ ) - a chain transfer agent to reduce molecular weight ${ }^{61}$ - were added subsequently. The vial was then degassed and sealed. The reaction was heated at $60{ }^{\circ} \mathrm{C}$ for one day, and at $80{ }^{\circ} \mathrm{C}$ for another two days. After cooling down to room temperature, $2 \mathrm{~mL}$ of THF was added to dissolve the polymer, followed by precipitation with $50 \mathrm{~mL}$ of deionized water. The precipitate was later washed with methanol and dried under vacuum overnight.

Zwitterion-functionalized polymer syntheses. Amine precursor polymer (400 mg, 0.18-0.30 mmol of amine group depending on the polymer) was dissolved in $8 \mathrm{~mL}$ THF and heated to 60 ${ }^{\circ} \mathrm{C}$. After $30 \mathrm{~min}$, an excess of sultone (1,4-butanesultone $(400 \mathrm{mg}, 2.94 \mathrm{mmol})$ or 1,3propanesultone $(400 \mathrm{mg}, 3.28 \mathrm{mmol})$ ) was added dropwise. $3 \mathrm{~mL}$ of methanol was added after 1 hour and another $3 \mathrm{~mL}$ of methanol was added after $24 \mathrm{hrs}$. The reaction was further heated at 60 ${ }^{\circ} \mathrm{C}$ for another day before cooling down to room temperature. The solution was transferred into a dialysis tube (molecular weight cut-off $=1000$ ) and stirred in $1000 \mathrm{~mL}$ of methanol for $2 \mathrm{hrs}$ and $1000 \mathrm{~mL}$ ethyl acetate for an additional $2 \mathrm{hrs}$. After dialysis, the solution inside the dialysis tube was transferred to a round-bottom flask and concentrated under vacuum. Clear transparent solids were obtained and dried under vacuum to remove residual solvents. The zwitterionic polymers are soluble in most common organic solvents, including green organic solvents such as ethyl and butyl acetate ${ }^{86}$ that are common antisolvents for perovskite $\mathrm{NC}$ purification. ${ }^{1,} 3,68{ }^{1} \mathrm{H}-\mathrm{NMR}$ spectra of the polymers synthesized here are provided in the Supporting Information.

Nanocrystal synthesis and purification. Preparation of $\mathrm{Pb}^{2+}$-oleate solution. $\mathrm{Pb}(\mathrm{OAc})_{2} \cdot 3 \mathrm{H}_{2} \mathrm{O}$ (1152 mg, $\left.3 \mathrm{mmol}\right)$, OA (1720 mg, $\left.\sim 6 \mathrm{mmol}\right)$, and ODE (3280 mg, $\left.4.15 \mathrm{~mL}\right)$ were combined in a $100 \mathrm{~mL}$ three neck flask. The temperature of the flask was slowly elevated and was held at $120{ }^{\circ} \mathrm{C}$ for $1 \mathrm{hr}$ on a Schlenk line under vacuum. The solution was then heated under $\mathrm{N}_{2}$ at $150{ }^{\circ} \mathrm{C}$ for $1 \mathrm{hr}$ to ensure that all reactants were well dissolved. The resulting $\sim 0.5 \mathrm{M}$ $\mathrm{Pb}^{2+}$ oleate solution $\left(\mathrm{Pb}(\mathrm{OA})_{2}\right)$ was transferred into a $\mathrm{N}_{2}$-filled glovebox and became a waxy solid when cooled to room temperature.

Preparation of $\mathrm{Cs}^{+}$-oleate solution. $\mathrm{Cs}_{2} \mathrm{CO}_{3}$ (1628 $\mathrm{mg}, 10 \mathrm{mmol}$ of $\mathrm{Cs}^{+}$ions), OA (4500 mg, $\sim 15 \mathrm{mmol})$, and ODE (15 g, $20 \mathrm{~mL})$ were combined in a $100 \mathrm{~mL}$ three neck flask. The solution was initially degassed on a Schlenk line at room temperature to remove highly volatile compounds. The temperature was then slowly elevated and held at $120{ }^{\circ} \mathrm{C}$ for $1 \mathrm{hr}$ under vacuum. The solution was then heated under $\mathrm{N}_{2}$ to $150{ }^{\circ} \mathrm{C}$ for $1 \mathrm{hr}$ to ensure that all reactants were well dissolved. The resulting $\sim 0.5 \mathrm{M} \mathrm{Cs}^{+}$oleate solution (CsOA) was transferred into a $\mathrm{N}_{2-}$ filled glovebox and became a highly viscous, turbid liquid when cooled to room temperature.

$C s P b\left(B_{1-x} I_{x}\right)_{3} N C$ synthesis. Perovskite NCs were prepared using a procedure adapted from 
the literature. ${ }^{68,92} \mathrm{CsOA}$ and $\mathrm{Pb}(\mathrm{OA})_{2}$ precursor solutions were heated to $130{ }^{\circ} \mathrm{C}$ in a $\mathrm{N}_{2}$ filled glovebox to fully dissolve all of their components. Once dissolved, CsOA $(0.8 \mathrm{~mL}, \sim 0.4 \mathrm{mmol}$ $\left.\mathrm{Cs}^{+}\right)$and $\mathrm{Pb}(\mathrm{OA})_{2}\left(1 \mathrm{~mL}, \sim 0.5 \mathrm{mmol} \mathrm{Pb}^{2+}\right)$ were loaded into a $100 \mathrm{~mL}$ three neck flask. The flask was removed from the glovebox and ODE (6650 mg, $8.5 \mathrm{~mL})$ and OAm (420 mg, 1.5 mmol) were loaded into the flask. The resulting metal oleate solution was degassed on a Schlenk line at $120{ }^{\circ} \mathrm{C}$ until the base pressure of the line was reached (typically 1-2 hrs). A solution containing TMS-Br $(180 \mu \mathrm{L}, 1.4 \mathrm{mmol})$ (or TMS-I $(200 \mu \mathrm{L}, 1.4 \mathrm{mmol}))$ and $400 \mu \mathrm{L}$ of ODE was prepared and sealed with a septum in a pear-shaped flask in a $\mathrm{N}_{2}$-filled glovebox. The metal oleate solution on the Schlenk line was heated under $\mathrm{N}_{2}$ to $180^{\circ} \mathrm{C}$. Once the injection temperature was reached, the TMS-Br (or TMS-I) solution was swiftly injected into the flask. The reaction was quenched after $\sim 5 \mathrm{~s}$ with an ice bath and the resulting $\mathrm{CsPb}\left(\mathrm{Br}_{1-x} \mathrm{I}_{x}\right)_{3} \mathrm{NCs}$ were centrifuged from the crude reaction solution at $1318 \mathrm{gx}$ for $10 \mathrm{~min}$. The precipitate was resuspended in hexanes and the resulting solution was precipitated with an excess of anhydrous ethyl acetate. This turbid solution was centrifuged at $1318 \mathrm{gx}$ for $15 \mathrm{~min}$ and the precipitate was resuspended in hexanes. The hexanes solution was again centrifuged at $1318 \mathrm{gx}$ for $5 \mathrm{~min}$ and the supernatant was dried under $\mathrm{N}_{2}$ gas flow and transferred into a $\mathrm{N}_{2}$-filled glovebox. Once in the glovebox, the $\mathrm{CsPb}\left(\mathrm{Br}_{1}\right.$ $\left.{ }_{x} \mathrm{I}_{x}\right)_{3} \mathrm{NC}$ pellet was resuspended in hexanes and the solution was filtered through a $0.22 \mu \mathrm{m}$ polytetrafluoroethylene (PTFE) syringe filter. Anion-alloyed $\operatorname{CsPb}\left(\mathrm{Br}_{1-x} \mathrm{I}_{x}\right)_{3} \quad \mathrm{NCs}$ were

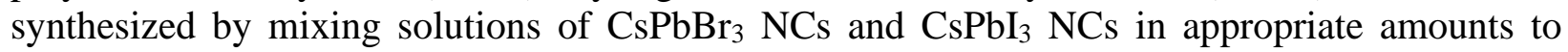
obtain a solution with a PL max of $\sim 660 \mathrm{~nm}$. Solution-state absorption spectra of the undoped NCs synthesized here are provided in the Supporting Information.

$\mathrm{Yb}^{3+}: \mathrm{CsPCl}_{3} \mathrm{NC}$ synthesis. NCs exchanged into ZFP3 were prepared using methods reported previously. ${ }^{9}$ Zwitterion-capped $\mathrm{Yb}^{3+}: \mathrm{CsPbCl}_{3} \mathrm{NCs}$ were synthesized according to the same procedure, but OAm was replaced with $100 \mathrm{mg} \mathrm{ZW}$-lig and the washed NCs were suspended in toluene rather than hexanes.

General aspects of NC/polymer composite film preparation. Glass substrates were cut and sonicated for 15 min sequentially in deionized water with $2 \%$ detergent, deionized water, acetone, and 2-propanol; after which each substrate was dried with compressed air. To transfer perovskite NCs into a zwitterionic polymer matrix, the desired quantities of polymer were added to $0.75 \mathrm{~mL}$ of ethyl acetate in a $\mathrm{N}_{2}$-filled glovebox and stirred overnight. Then, a small volume of NC solution in hexanes was added to the solution of zwitterionic polymer in ethyl acetate. NC concentrations were determined using literature perovskite NC extinction coefficients, ${ }^{60}$ and the volume of $\mathrm{NC}$ solution added was determined such that the equivalents (Eq) of zwitterionic groups per NC was kept between 10 and $300 \mathrm{kEq}$. This solution was then removed from the glovebox and sonicated for $5 \mathrm{~min}$. The resulting NC/zwitterionic polymer composites were precipitated out of solution by adding an excess of hexanes and centrifuging the resulting turbid solution at $1318 \mathrm{gx}$ for $10 \mathrm{~min}$. Very few NCs remained in the resulting supernatant (see Supporting Information), indicating that the native aliphatic ligands were successfully exchanged for the zwitterionic polymer ligands. This final pellet was then dispersed in $0.5 \mathrm{~mL}$ of butyl acetate. To investigate the effect of aggregates on the observed PL red shifts, we diluted a representative solution of $\mathrm{CsPbBr}_{3} \mathrm{NC} / \mathrm{ZP} 3$ composite by a factor of 50 with butyl acetate and drop cast an aliquot of this solution and all subsequent solutions onto clean glass substrates. After casting the first film, the remaining dilute NC/ZP3 solution was centrifuged at 16,060 gx for 10 minutes and the precipitate was discarded. Finally, the remaining centrifuged solution was filtered through a $0.22 \mu \mathrm{m}$ PTFE syringe filter. This process yielded a total of three solid samples for future characterization. 
PMMA films were fabricated from dispersions prepared in the following manner: $120 \mathrm{mg}$ of PMMA was capped and stirred in $0.45 \mathrm{~mL}$ of toluene overnight in an $\mathrm{N}_{2}$-filled glovebox. NCs were then added to the $25 \mathrm{wt} \%$ PMMA solution and stirred into the solution for several hours until a uniform dispersion was obtained. This solution was then drop cast onto a glass substrate in the $\mathrm{N}_{2}$-filled glovebox and allowed to dry for several hours before the resulting solid sample was exposed to ambient conditions. EVA lamination was performed with a Bent River SPL2828PIN laminator as follows: After a zwitterionic polymer or PMMA sample was drop cast and dried, a piece of EVA was placed between the dried sample and second clean glass substrate. This stack was placed on the laminator bed and was vacuum laminated at $80{ }^{\circ} \mathrm{C}$ for 10 min using a time-varying pressure profile with 10.4 psi of maximum applied pressure. The edges of the final samples were trimmed with a razor blade.

\section{Acknowledgments}

This research was primarily supported by the National Science Foundation (NSF) through the UW Molecular Engineering Materials Center, a Materials Research Science and Engineering Center (DMR-1719797 to DRG and CKL). This work was also supported by the State of Washington through the Washington Research Foundation (to JDM) and the Clean Energy Institute (to TAC and JDM). Part of this work was conducted at the Washington Clean Energy Testbeds and the Photonic Research Center, facilities operated by the University of Washington Clean Energy Institute. Part of this work was conducted at the Molecular Analysis Facility, a National Nanotechnology Coordinated Infrastructure site at the University of Washington that is supported in part by the National Science Foundation (ECC-1542101), the University of Washington, the Molecular Engineering \& Sciences Institute, and the Clean Energy Institute. YH thanks the Data Intensive Research Enabling Clean Technology (DIRECT) NSF National Research Traineeship (DGE-1633216) for support. We gratefully acknowledge the assistance of $\mathrm{K}$. Kluherz and E. Lavoie in collecting TEM images, S. Young for assistance collecting XRD data, and D. Kroupa for assistance collecting NIR PL data. We also acknowledge C. Krajewska for providing $\mathrm{Yb}^{3+}: \mathrm{CsPbl}_{3} \mathrm{NCs}$ that were used in early iterations of this work.

Supporting Information: The Supporting Information is available free of charge at http://pubs.acs.org.

Additional description of spectroscopic methods, ${ }^{1} \mathrm{H}-\mathrm{NMR}$ spectra of as-synthesized zwitterionic polymers, absorption and PL spectra of as-synthesized $\mathrm{CsPbBr}_{3}$ and $\mathrm{CsPbI}_{3} \mathrm{NCs}_{3}$ in solution, PLQYs as a function of absorption percentage for $\mathrm{CsPbBr}_{3} \mathrm{NCs}$ with and without zwitterionic polymer stabilization, additional absorption and PL spectra of solid-state $\mathrm{CsPbBr}_{3} \mathrm{NC}$ samples, PL decay curves for $\mathrm{CsPbr}_{3}$ and $\mathrm{CsPbI}_{3} \mathrm{NCs}, \mathrm{XRD}$ data of $\mathrm{CsPbBr}_{3}$ and $\mathrm{CsPbI}_{3} \mathrm{NCs}$, additional data from $\mathrm{CsPbBr}_{3}$ and $\mathrm{CsPbI}_{3} \mathrm{NC}$ samples under irradiation, $\mathrm{PL}$ spectra of $\mathrm{CsPb}\left(\mathrm{Br}_{1-x} \mathrm{I}_{x}\right)_{3} \mathrm{NCs}$ in a solid-state $\mathrm{ZP} 4$ composite, and a summary of publications reporting high solid-state $\mathrm{CsPbI}_{3} \mathrm{NC}$ PLQYs.

\section{References}

1. Protesescu, L.; Yakunin, S.; Bodnarchuk, M. I.; Krieg, F.; Caputo, R.; Hendon, C. H.; Yang, R. X.; Walsh, A.; Kovalenko, M. V. Nanocrystals of Cesium Lead Halide Perovskites $\left(\mathrm{CsPbX}_{3}, \mathrm{X}=\mathrm{Cl}, \mathrm{Br}\right.$, and I): Novel Optoelectronic Materials Showing Bright Emission with Wide Color Gamut. Nano Lett. 2015, 15, 3692-3696. 
2. Koscher, B. A.; Swabeck, J. K.; Bronstein, N. D.; Alivisatos, A. P. Essentially Trap-Free $\mathrm{CsPbBr}_{3}$ Colloidal Nanocrystals by Postsynthetic Thiocyanate Surface Treatment. J. Am. Chem. Soc. 2017, 139, 6566-6569.

3. Zhang, Y.; Siegler, T. D.; Thomas, C. J.; Abney, M. K.; Shah, T.; De Gorostiza, A.; Greene, R. M.; Korgel, B. A. A "Tips and Tricks" Practical Guide to the Synthesis of Metal Halide Perovskite Nanocrystals. Chem. Mater. 2020, 32, 5410-5423.

4. Pradhan, N. Tips and Twists in Making High Photoluminescence Quantum Yield Perovskite Nanocrystals. ACS Energy Lett. 2019, 4, 1634-1638.

5. Utzat, H.; Shulenberger, K. E.; Achorn, O. B.; Nasilowski, M.; Sinclair, T. S.; Bawendi, M. G. Probing Linewidths and Biexciton Quantum Yields of Single Cesium Lead Halide Nanocrystals in Solution. Nano Lett. 2017, 17, 6838-6846.

6. Creutz, S. E.; Crites, E. N.; De Siena, M. C.; Gamelin, D. R. Anion Exchange in Cesium Lead Halide Perovskite Nanocrystals and Thin Films Using Trimethylsilyl Halide Reagents. Chem. Mater. 2018, 30, 4887-4891.

7. Nedelcu, G.; Protesescu, L.; Yakunin, S.; Bodnarchuk, M. I.; Grotevent, M. J.; Kovalenko, M. V. Fast Anion-Exchange in Highly Luminescent Nanocrystals of Cesium Lead Halide Perovskites $\left(\mathrm{CsPbX}_{3}, \mathrm{X}=\mathrm{Cl}, \mathrm{Br}, \mathrm{I}\right)$. Nano Lett. 2015, 15, 5635-5640.

8. Imran, M.; Caligiuri, V.; Wang, M.; Goldoni, L.; Prato, M.; Krahne, R.; De Trizio, L.; Manna, L. Benzoyl Halides as Alternative Precursors for the Colloidal Synthesis of LeadBased Halide Perovskite Nanocrystals. J. Am. Chem. Soc. 2018, 140, 2656-2664.

9. Milstein, T. J.; Kroupa, D. M.; Gamelin, D. R. Picosecond Quantum Cutting Generates Photoluminescence Quantum Yields Over 100\% in Ytterbium-Doped $\mathrm{CsPbCl}_{3} \mathrm{Nanocrystals}$ Nano Lett. 2018, 18, 3792-3799.

10. Liu, W.; Lin, Q.; Li, H.; Wu, K.; Robel, I.; Pietryga, J. M.; Klimov, V. I. Mn ${ }^{2+}$-Doped Lead Halide Perovskite Nanocrystals with Dual-Color Emission Controlled by Halide Content. $J$. Am. Chem. Soc. 2016, 138, 14954-14961.

11. Pan, G.; Bai, X.; Yang, D.; Chen, X.; Jing, P.; Qu, S.; Zhang, L.; Zhou, D.; Zhu, J.; Xu, W., et al. Doping Lanthanide into Perovskite Nanocrystals: Highly Improved and Expanded Optical Properties. Nano Lett. 2017, 17, 8005-8011.

12. Zhou, D.; Liu, D.; Pan, G.; Chen, X.; Li, D.; Xu, W.; Bai, X.; Song, H. Cerium and Ytterbium Codoped Halide Perovskite Quantum Dots: A Novel and Efficient Downconverter for Improving the Performance of Silicon Solar Cells. Adv. Mater. 2017, 29, 1704149.

13. De Siena, M. C.; Sommer, D. E.; Creutz, S. E.; Dunham, S. T.; Gamelin, D. R. Spinodal Decomposition During Anion Exchange in Colloidal $\mathrm{Mn}^{2+}$-Doped $\mathrm{CsPbX}_{3}(\mathrm{X}=\mathrm{Cl}, \mathrm{Br})$ Perovskite Nanocrystals. Chem. Mater. 2019, 31, 7711-7722.

14. Becker, M. A.; Vaxenburg, R.; Nedelcu, G.; Sercel, P. C.; Shabaev, A.; Mehl, M. J.; Michopoulos, J. G.; Lambrakos, S. G.; Bernstein, N.; Lyons, J. L., et al. Bright Triplet Excitons in Caesium Lead Halide Perovskites. Nature 2018, 553, 189-193.

15. Isarov, M.; Tan, L. Z.; Bodnarchuk, M. I.; Kovalenko, M. V.; Rappe, A. M.; Lifshitz, E. Rashba Effect in a Single Colloidal $\mathrm{CsPbBr}_{3}$ Perovskite Nanocrystal Detected by MagnetoOptical Measurements. Nano Lett. 2017, 17, 5020-5026.

16. Liu, Z.; Vaswani, C.; Yang, X.; Zhao, X.; Yao, Y.; Song, Z.; Cheng, D.; Shi, Y.; Luo, L.; Mudiyanselage, D. H., et al. Ultrafast Control of Excitonic Rashba Fine Structure by Phonon Coherence in the Metal Halide Perovskite $\mathrm{CH}_{3} \mathrm{NH}_{3} \mathrm{PbI}_{3}$. Phys. Rev. Lett. 2020, 124, 157401. 
17. Akkerman, Q. A.; Rainò, G.; Kovalenko, M. V.; Manna, L. Genesis, Challenges and Opportunities for Colloidal Lead Halide Perovskite Nanocrystals. Nat. Mater. 2018, 17, 394405.

18. Nenon, D. P.; Pressler, K.; Kang, J.; Koscher, B. A.; Olshansky, J. H.; Osowiecki, W. T.; Koc, M. A.; Wang, L.-W.; Alivisatos, A. P. Design Principles for Trap-Free CsPbX 3 Nanocrystals: Enumerating and Eliminating Surface Halide Vacancies with Softer Lewis Bases. J. Am. Chem. Soc. 2018, 140, 17760-17772.

19. Kang, J.; Wang, L.-W. High Defect Tolerance in Lead Halide Perovskite CsPbBr 3 . J. Phys. Chem. Lett. 2017, 8, 489-493.

20. ten Brinck, S.; Zaccaria, F.; Infante, I. Defects in Lead Halide Perovskite Nanocrystals: Analogies and (Many) Differences with the Bulk. ACS Energy Lett. 2019, 4, 2739-2747.

21. Sun, C.; Zhang, Y.; Ruan, C.; Yin, C.; Wang, X.; Wang, Y.; Yu, W. W. Efficient and Stable White LEDs with Silica-Coated Inorganic Perovskite Quantum Dots. Adv. Mater. 2016, 28, 10088-10094.

22. Zhou, Q.; Bai, Z.; Lu, W.-g.; Wang, Y.; Zou, B.; Zhong, H. In situ Fabrication of Halide Perovskite Nanocrystal-Embedded Polymer Composite Films with Enhanced Photoluminescence for Display Backlights. Adv. Mater. 2016, 28, 9163-9168.

23. Yakunin, S.; Protesescu, L.; Krieg, F.; Bodnarchuk, M. I.; Nedelcu, G.; Humer, M.; Luca, G. D.; Fiebig, M.; Heiss, W.; Kovalenko, M. V. Low-Threshold Amplified Spontaneous Emission and Lasing from Colloidal Nanocrystals of Caesium Lead Halide Perovskites. Nat. Commun. 2015, 6, 8056.

24. Makarov, N. S.; Guo, S.; Isaienko, O.; Liu, W.; Robel, I.; Klimov, V. I. Spectral and Dynamical Properties of Single Excitons, Biexcitons, and Trions in Cesium-Lead-Halide Perovskite Quantum Dots. Nano Lett. 2016, 16, 2349-2362.

25. Huang, C.-Y.; Zou, C.; Mao, C.; Corp, K. L.; Yao, Y.-C.; Lee, Y.-J.; Schlenker, C. W.; Jen, A. K. Y.; Lin, L. Y. CsPbBr 3 Perovskite Quantum Dot Vertical Cavity Lasers with Low Threshold and High Stability. ACS Photonics 2017, 4, 2281-2289.

26. Wang, Y.; Zhi, M.; Chang, Y.-Q.; Zhang, J.-P.; Chan, Y. Stable, Ultralow Threshold Amplified Spontaneous Emission from $\mathrm{CsPbBr}_{3}$ Nanoparticles Exhibiting Trion Gain. Nano Lett. 2018, 18, 4976-4984.

27. Park, Y.-S.; Guo, S.; Makarov, N. S.; Klimov, V. I. Room Temperature Single-Photon Emission from Individual Perovskite Quantum Dots. ACS Nano 2015, 9, 10386-10393.

28. Rainò, G.; Nedelcu, G.; Protesescu, L.; Bodnarchuk, M. I.; Kovalenko, M. V.; Mahrt, R. F.; Stöferle, T. Single Cesium Lead Halide Perovskite Nanocrystals at Low Temperature: Fast Single-Photon Emission, Reduced Blinking, and Exciton Fine Structure. ACS Nano 2016, 10, 2485-2490.

29. Utzat, H.; Sun, W.; Kaplan, A. E. K.; Krieg, F.; Ginterseder, M.; Spokoyny, B.; Klein, N. D.; Shulenberger, K. E.; Perkinson, C. F.; Kovalenko, M. V., et al. Coherent Single-Photon Emission from Colloidal Lead Halide Perovskite Quantum Dots. Science 2019, 363, 10681072.

30. Meinardi, F.; Akkerman, Q. A.; Bruni, F.; Park, S.; Mauri, M.; Dang, Z.; Manna, L.; Brovelli, S. Doped Halide Perovskite Nanocrystals for Reabsorption-Free Luminescent Solar Concentrators. ACS Energy Lett. 2017, 2, 2368-2377.

31. Zhao, H.; Benetti, D.; Tong, X.; Zhang, H.; Zhou, Y.; Liu, G.; Ma, D.; Sun, S.; Wang, Z. M.; Wang, Y., et al. Efficient and Stable Tandem Luminescent Solar Concentrators based on Carbon Dots and Perovskite Quantum Dots. Nano Energy 2018, 50, 756-765. 
32. Cohen, T. A.; Milstein, T. J.; Kroupa, D. M.; MacKenzie, J. D.; Luscombe, C. K.; Gamelin, D. R. Quantum-Cutting $\mathrm{Yb}^{3+}$-Doped Perovskite Nanocrystals for Monolithic Bilayer Luminescent Solar Concentrators. J. Mater. Chem. A 2019, 7, 9279-9288.

33. Wei, M.; Arquer, F. P. G. d.; Walters, G.; Yang, Z.; Quan, L. N.; Kim, Y.; Sabatini, R.; Quintero-Bermudez, R.; Gao, L.; Fan, J. Z., et al. Ultrafast Narrowband Exciton Routing within Layered Perovskite Nanoplatelets Enables Low-Loss Luminescent Solar Concentrators. Nat. Energy 2019, 4, 197-205.

34. Yuan, G.; Ritchie, C.; Ritter, M.; Murphy, S.; Gómez, D. E.; Mulvaney, P. The Degradation and Blinking of Single $\mathrm{CsPbI}_{3}$ Perovskite Quantum Dots. J. Phys. Chem. C 2018, 122, 13407-13415.

35. Vicente, J. R.; Rafiei Miandashti, A.; Sy Piecco, K. W. E.; Pyle, J. R.; Kordesch, M. E.; Chen, J. Single-Particle Organolead Halide Perovskite Photoluminescence as a Probe for Surface Reaction Kinetics. ACS Appl. Mater. Interfaces 2019, 11, 18034-18043.

36. Meyns, M.; Perálvarez, M.; Heuer-Jungemann, A.; Hertog, W.; Ibáñez, M.; Nafria, R.; Genç, A.; Arbiol, J.; Kovalenko, M. V.; Carreras, J., et al. Polymer-Enhanced Stability of Inorganic Perovskite Nanocrystals and Their Application in Color Conversion LEDs. ACS Appl. Mater. Interfaces 2016, 8, 19579-19586.

37. Raja, S. N.; Bekenstein, Y.; Koc, M. A.; Fischer, S.; Zhang, D.; Lin, L.; Ritchie, R. O.; Yang, P.; Alivisatos, A. P. Encapsulation of Perovskite Nanocrystals into Macroscale Polymer Matrices: Enhanced Stability and Polarization. ACS Appl. Mater. Interfaces 2016, 8, 3552335533.

38. Wu, H.; Wang, S.; Cao, F.; Zhou, J.; Wu, Q.; Wang, H.; Li, X.; Yin, L.; Yang, X. Ultrastable Inorganic Perovskite Nanocrystals Coated with a Thick Long-Chain Polymer for Efficient White Light-Emitting Diodes. Chem. Mater. 2019, 31, 1936-1940.

39. Wei, S.; Zhu, H.; Zhang, J.; Wang, L.; An, M.; Wang, Y.; Zhang, X.; Liu, Y. Luminescent Perovskite Nanocrystal-Epoxy Resin Composite with High Stability Against Water and Air. J. Alloys Compd. 2019, 789, 209-214.

40. Tong, J.; Wu, J.; Shen, W.; Zhang, Y.; Liu, Y.; Zhang, T.; Nie, S.; Deng, Z. Direct HotInjection Synthesis of Lead Halide Perovskite Nanocubes in Acrylic Monomers for Ultrastable and Bright Nanocrystal-Polymer Composite Films. ACS Appl. Mater. Interfaces 2019, 11, 9317-9325.

41. Wang, H.-C.; Lin, S.-Y.; Tang, A.-C.; Singh, B. P.; Tong, H.-C.; Chen, C.-Y.; Lee, Y.-C.; Tsai, T.-L.; Liu, R.-S. Mesoporous Silica Particles Integrated with All-Inorganic CsPbBr 3 Perovskite Quantum-Dot Nanocomposites (MP-PQDs) with High Stability and Wide Color Gamut Used for Backlight Display. Angew. Chem. Int. Ed. 2016, 55, 7924-7929.

42. Chen, D.; Fang, G.; Chen, X. Silica-Coated Mn-Doped $\mathrm{CsPb}(\mathrm{Cl} / \mathrm{Br})_{3}$ Inorganic Perovskite Quantum Dots: Exciton-to-Mn Energy Transfer and Blue-Excitable Solid-State Lighting. ACS Appl. Mater. Interfaces 2017, 9, 40477-40487.

43. Li, Z.; Kong, L.; Huang, S.; Li, L. Highly Luminescent and Ultrastable $\mathrm{CsPbBr}_{3}$ Perovskite Quantum Dots Incorporated into a Silica/Alumina Monolith. Angew. Chem. 2017, 129, 82468250.

44. Pramanik, A.; Gates, K.; Patibandla, S.; Davis, D.; Begum, S.; Iftekhar, R.; Alamgir, S.; Paige, S.; Porter, M. M.; Ray, P. C. Water-Soluble and Bright Luminescent Cesium-LeadBromide Perovskite Quantum Dot-Polymer Composites for Tumor-Derived Exosome Imaging. ACS Appl. Bio Mater. 2019, 2, 5872-5879. 
45. Lu, Z.; Li, Y.; Qiu, W.; Rogach, A. L.; Nagl, S. Composite Films of CsPbBr 3 Perovskite Nanocrystals in a Hydrophobic Fluoropolymer for Temperature Imaging in Digital Microfluidics. ACS Appl. Mater. Interfaces 2020, 12, 19805-19812.

46. Ahmed, T.; Seth, S.; Samanta, A. Boosting the Photoluminescence of $\mathrm{CsPbX}_{3}(\mathrm{X}=\mathrm{Cl}, \mathrm{Br}, \mathrm{I})$ Perovskite Nanocrystals Covering a Wide Wavelength Range by Postsynthetic Treatment with Tetrafluoroborate Salts. Chem. Mater. 2018, 30, 3633-3637.

47. Zhang, Q.; Li, Z.; Liu, M.; Kong, L.; Zheng, W.; Wang, B.; Li, L. Bifunctional Passivation Strategy to Achieve Stable $\mathrm{CsPBr}_{3}$ Nanocrystals with Drastically Reduced ThermalQuenching. J. Phys. Chem. Lett. 2020, 11, 993-999.

48. Sun, H.; Yang, Z.; Wei, M.; Sun, W.; Li, X.; Ye, S.; Zhao, Y.; Tan, H.; Kynaston, E. L.; Schon, T. B., et al. Chemically Addressable Perovskite Nanocrystals for Light-Emitting Applications. Adv. Mater. 2017, 29, 1701153.

49. Kim, H.; So, S.; Ribbe, A.; Liu, Y.; Hu, W.; Duzhko, V. V.; Hayward, R. C.; Emrick, T. Functional Polymers for Growth and Stabilization of $\mathrm{CsPbBr}_{3}$ Perovskite Nanoparticles. Chem. Commun. 2019, 55, 1833-1836.

50. Suh, Y.-H.; Kim, T.; Choi, J. W.; Lee, C.-L.; Park, J. High-Performance CsPbX 3 Perovskite Quantum-Dot Light-Emitting Devices via Solid-State Ligand Exchange. ACS Appl. Nano Mater. 2018, 1, 488-496.

51. He, Y.; Yoon, Y. J.; Harn, Y. W.; Biesold-McGee, G. V.; Liang, S.; Lin, C. H.; Tsukruk, V. V.; Thadhani, N.; Kang, Z.; Lin, Z. Unconventional Route to Dual-Shelled Organolead Halide Perovskite Nanocrystals with Controlled Dimensions, Surface Chemistry, and Stabilities. Sci. Adv. 2019, 5, eaax4424.

52. Hou, S.; Guo, Y.; Tang, Y.; Quan, Q. Synthesis and Stabilization of Colloidal Perovskite Nanocrystals by Multidentate Polymer Micelles. ACS Appl. Mater. Interfaces 2017, 9, 18417-18422.

53. Hui, L. S.; Beswick, C.; Getachew, A.; Heilbrunner, H.; Liang, K.; Hanta, G.; Arbi, R.; Munir, M.; Dawood, H.; Isik Goktas, N., et al. Reverse Micelle Templating Route to Ordered Monodispersed Spherical Organo-Lead Halide Perovskite Nanoparticles for Light Emission. ACS Appl. Nano Mater. 2019, 2, 4121-4132.

54. Liu, Y.; Wang, Z.; Liang, S.; Li, Z.; Zhang, M.; Li, H.; Lin, Z. Polar Organic SolventTolerant Perovskite Nanocrystals Permanently Ligated with Polymer Hairs via Star-Like Molecular Bottlebrush Trilobe Nanoreactors. Nano Lett. 2019, 19, 9019-9028.

55. Xin, Y.; Shen, W.; Deng, Z.; Zhang, J. Highly Emissive and Color-Tunable Perovskite Cross-Linkers for Luminescent Polymer Networks. ACS Appl. Mater. Interfaces 2018, 10, 28971-28978.

56. Guan, H.; Zhao, S.; Wang, H.; Yan, D.; Wang, M.; Zang, Z. Room Temperature Synthesis of Stable Single Silica-Coated $\mathrm{CsPbBr}_{3}$ Quantum Dots Combining Tunable Red Emission of Ag-In-Zn-S for High-CRI White Light-Emitting Diodes. Nano Energy 2020, 67, 104279.

57. Jancik Prochazkova, A.; Demchyshyn, S.; Yumusak, C.; Másilko, J.; Brüggemann, O.; Weiter, M.; Kaltenbrunner, M.; Sariciftci, N. S.; Krajcovic, J.; Salinas, Y., et al. Proteinogenic Amino Acid Assisted Preparation of Highly Luminescent Hybrid Perovskite Nanoparticles. ACS Appl. Nano Mater. 2019, 2, 4267-4274.

58. Pan, A.; Wang, J.; Jurow, M. J.; Jia, M.; Liu, Y.; Wu, Y.; Zhang, Y.; He, L.; Liu, Y. General Strategy for the Preparation of Stable Luminous Nanocomposite Inks Using Chemically Addressable $\mathrm{CsPbX}_{3}$ Peroskite Nanocrystals. Chem. Mater. 2018, 30, 2771-2780. 
59. Liang, P.; Zhang, P.; Pan, A.; Yan, K.; Zhu, Y.; Yang, M.; He, L. Unusual Stability and Temperature-Dependent Properties of Highly Emissive $\mathrm{CsPbBr}_{3}$ Perovskite Nanocrystals Obtained from in situ Crystallization in Poly(vinylidene difluoride). ACS Appl. Mater. Interfaces 2019, 11, 22786-22793.

60. De Roo, J.; Ibáñez, M.; Geiregat, P.; Nedelcu, G.; Walravens, W.; Maes, J.; Martins, J. C.; Van Driessche, I.; Kovalenko, M. V.; Hens, Z. Highly Dynamic Ligand Binding and Light Absorption Coefficient of Cesium Lead Bromide Perovskite Nanocrystals. ACS Nano 2016, 10, 2071-2081.

61. Çetinkaya, O.; Demirci, G.; Mergo, P. Effect of the different chain transfer agents on molecular weight and optical properties of poly(methyl methacrylate). Opt. Mater. 2017, 70, 25-30.

62. Liu, F.; Zhang, Y.; Ding, C.; Kobayashi, S.; Izuishi, T.; Nakazawa, N.; Toyoda, T.; Ohta, T.; Hayase, S.; Minemoto, T., et al. Highly Luminescent Phase-Stable $\mathrm{CsPbI}_{3}$ Perovskite Quantum Dots Achieving Near 100\% Absolute Photoluminescence Quantum Yield. ACS Nano 2017, 11, 10373-10383.

63. Wu, L.; Zhong, Q.; Yang, D.; Chen, M.; Hu, H.; Pan, Q.; Liu, H.; Cao, M.; Xu, Y.; Sun, B., et al. Improving the Stability and Size Tunability of Cesium Lead Halide Perovskite Nanocrystals Using Trioctylphosphine Oxide as the Capping Ligand. Langmuir 2017, 33, 12689-12696.

64. Tan, Y.; Zou, Y.; Wu, L.; Huang, Q.; Yang, D.; Chen, M.; Ban, M.; Wu, C.; Wu, T.; Bai, S., et al. Highly Luminescent and Stable Perovskite Nanocrystals with Octylphosphonic Acid as a Ligand for Efficient Light-Emitting Diodes. ACS Appl. Mater. Interfaces 2018, 10, 37843792.

65. Zhang, B.; Goldoni, L.; Zito, J.; Dang, Z.; Almeida, G.; Zaccaria, F.; de Wit, J.; Infante, I.; De Trizio, L.; Manna, L. Alkyl Phosphonic Acids Deliver $\mathrm{CsPbBr}_{3}$ Nanocrystals with High Photoluminescence Quantum Yield and Truncated Octahedron Shape. Chem. Mater. 2019, $31,9140-9147$.

66. Wu, J.; Tong, J.; Gao, Y.; Wang, A.; Zhang, T.; Tan, H.; Nie, S.; Deng, Z. Efficient and Stable Thin-Film Luminescent Solar Concentrators Enabled by Near-Infrared Emission Perovskite Nanocrystals. Angew. Chem. Int. Ed. 2020, 59, 7738-7742.

67. Krieg, F.; Ong, Q. K.; Burian, M.; Rainò, G.; Naumenko, D.; Amenitsch, H.; Süess, A.; Grotevent, M. J.; Krumeich, F.; Bodnarchuk, M. I., et al. Stable Ultraconcentrated and Ultradilute Colloids of $\mathrm{CsPbX}_{3}(\mathrm{X}=\mathrm{Cl}, \mathrm{Br})$ Nanocrystals Using Natural Lecithin as a Capping Ligand. J. Am. Chem. Soc. 2019, 141, 19839-19849.

68. Krieg, F.; Ochsenbein, S. T.; Yakunin, S.; ten Brinck, S.; Aellen, P.; Süess, A.; Clerc, B.; Guggisberg, D.; Nazarenko, O.; Shynkarenko, Y., et al. Colloidal CsPbX 3 (X = Cl, Br, I) Nanocrystals 2.0: Zwitterionic Capping Ligands for Improved Durability and Stability. ACS Energy Lett. 2018, 3, 641-646.

69. Chen, Q.; Yuan, L.; Duan, R.; Huang, P.; Fu, J.; Ma, H.; Wang, X.; Zhou, Y.; Song, B. Zwitterionic Polymer: A Facile Interfacial Material Works at Both Anode and Cathode in pi-n Perovskite Solar Cells. Sol. RRL 2019, 3, 1900118.

70. Zhou, S.; Zhu, T.; Zheng, L.; Zhang, D.; Xu, W.; Liu, L.; Cheng, G.; Zheng, J.; Gong, X. A Zwitterionic Polymer as an Interfacial Layer for Efficient and Stable Perovskite Solar Cells. RSC Advances 2019, 9, 30317-30324. 
71. Kim, H.; Hight - Huf, N.; Kang, J.-H.; Bisnoff, P.; Sundararajan, S.; Thompson, T.; Barnes, M.; Hayward, R. C.; Emrick, T. Polymer Zwitterions for Stabilization of $\mathrm{CsPbBr}_{3}$ Perovskite Nanoparticles and Nanocomposite Films. Angew. Chem. Int. Ed. 2020, 59, 10802-10806.

72. Wang, S.; Du, L.; Jin, Z.; Xin, Y.; Mattoussi, H. Enhanced Stabilization and Easy Phase Transfer of $\mathrm{CsPbBr}_{3}$ Perovskite Quantum Dots Promoted by High Affinity Polyzwitterionic Ligands. J. Am. Chem. Soc. 2020, 142, 12669-12680.

73. Cabanetos, C.; El Labban, A.; Bartelt, J. A.; Douglas, J. D.; Mateker, W. R.; Fréchet, J. M. J.; McGehee, M. D.; Beaujuge, P. M. Linear Side Chains in Benzo[1,2-b:4,5-b' ]dithiophene Thieno[3,4-c]pyrrole-4,6-dione Polymers Direct Self-Assembly and Solar Cell Performance. J. Am. Chem. Soc. 2013, 135, 4656-4659.

74. Gadisa, A.; Oosterbaan, W. D.; Vandewal, K.; Bolsée, J.-C.; Bertho, S.; D'Haen, J.; Lutsen, L.; Vanderzande, D.; Manca, J. V. Effect of Alkyl Side-Chain Length on Photovoltaic Properties of Poly(3-alkylthiophene)/PCBM Bulk Heterojunctions. Adv. Funct. Mater. 2009, 19, 3300-3306.

75. Xie, R.; Chen, Z.; Zhong, W.; Zhang, G.; Huang, Y.; Ying, L.; Huang, F.; Cao, Y. Synthesis and characterization of $\pi$-conjugated copolymers based on alkyltriazolyl substituted benzodithiophene. New J. Chem. 2016, 40, 4727-4734.

76. Meager, I.; Ashraf, R. S.; Mollinger, S.; Schroeder, B. C.; Bronstein, H.; Beatrup, D.; Vezie, M. S.; Kirchartz, T.; Salleo, A.; Nelson, J., et al. Photocurrent Enhancement from Diketopyrrolopyrrole Polymer Solar Cells through Alkyl-Chain Branching Point Manipulation. J. Am. Chem. Soc. 2013, 135, 11537-11540.

77. Shivhare, R.; Erdmann, T.; Hörmann, U.; Collado-Fregoso, E.; Zeiske, S.; Benduhn, J.; Ullbrich, S.; Hübner, R.; Hambsch, M.; Kiriy, A., et al. Alkyl Branching Position in Diketopyrrolopyrrole Polymers: Interplay between Fibrillar Morphology and Crystallinity and Their Effect on Photogeneration and Recombination in Bulk-Heterojunction Solar Cells. Chem. Mater. 2018, 30, 6801-6809.

78. Lei, T.; Dou, J.-H.; Pei, J. Influence of Alkyl Chain Branching Positions on the Hole Mobilities of Polymer Thin-Film Transistors. Adv. Mater. 2012, 24, 6457-6461.

79. Groh, W. Overtone Absorption in Macromolecules for Polymer Optical Fibers. Die Makromol. Chemie 1988, 189, 2861-2874.

80. Obrezkova, M. A.; Kalinina, A. A.; Pavlichenko, I. V.; Vasilenko, N. G.; Mironova, M. V.; Semakov, A. V.; Kulichikhin, V. G.; Buzin, M. I.; Muzafarov, A. M. Comb-Like Polymethylsiloxanes. Synthesis, Structure and Properties. Silicon 2015, 7, 177-189.

81. Knauf, R. R.; Lennox, J. C.; Dempsey, J. L. Quantifying Ligand Exchange Reactions at CdSe Nanocrystal Surfaces. Chem. Mater. 2016, 28, 4762-4770.

82. Dierick, R.; Van den Broeck, F.; De Nolf, K.; Zhao, Q.; Vantomme, A.; Martins, J. C.; Hens, Z. Surface Chemistry of CuInS 2 Colloidal Nanocrystals, Tight Binding of L-Type Ligands. Chem. Mater. 2014, 26, 5950-5957.

83. Hens, Z.; Martins, J. C. A Solution NMR Toolbox for Characterizing the Surface Chemistry of Colloidal Nanocrystals. Chem. Mater. 2013, 25, 1211-1221.

84. Imran, M.; Ijaz, P.; Goldoni, L.; Maggioni, D.; Petralanda, U.; Prato, M.; Almeida, G.; Infante, I.; Manna, L. Simultaneous Cationic and Anionic Ligand Exchange For Colloidally Stable CsPbBr3 Nanocrystals. ACS Energy Lett. 2019, 4, 819-824.

85. Quarta, D.; Imran, M.; Capodilupo, A.-L.; Petralanda, U.; van Beek, B.; De Angelis, F.; Manna, L.; Infante, I.; De Trizio, L.; Giansante, C. Stable Ligand Coordination at the Surface of Colloidal CsPbBr 3 Nanocrystals. J. Phys. Chem. Lett. 2019, 10, 3715-3726. 
86. Prat, D.; Wells, A.; Hayler, J.; Sneddon, H.; McElroy, C. R.; Abou-Shehada, S.; Dunn, P. J. CHEM21 Selection Guide of Classical- and Less Classical-Solvents. Green Chem. 2015, 18, 288-296.

87. Shimizu, K. T.; Böhmer, M.; Estrada, D.; Gangwal, S.; Grabowski, S.; Bechtel, H.; Kang, E.; Vampola, K. J.; Chamberlin, D.; Shchekin, O. B., et al. Toward Commercial Realization of Quantum Dot Based White Light-Emitting Diodes for General Illumination. Photonics Res. 2017, 5, A1-A6.

88. Sorensen, C. M. Light Scattering by Fractal Aggregates: A Review. Aerosol Sci. Tech. 2001, 35, 648-687.

89. Rainò, G.; Landuyt, A.; Krieg, F.; Bernasconi, C.; Ochsenbein, S. T.; Dirin, D. N.;

Bodnarchuk, M. I.; Kovalenko, M. V. Underestimated Effect of a Polymer Matrix on the Light Emission of Single $\mathrm{CsPBr}_{3}$ Nanocrystals. Nano Lett. 2019, 19, 3648-3653.

90. Di Stasio, F.; Christodoulou, S.; Huo, N.; Konstantatos, G. Near-Unity Photoluminescence Quantum Yield in $\mathrm{CsPbBr}_{3}$ Nanocrystal Solid-State Films via Postsynthesis Treatment with Lead Bromide. Chem. Mater. 2017, 29, 7663-7667.

91. Dutta, A.; Pradhan, N. Phase-Stable Red-Emitting $\mathrm{CsPbI}_{3}$ Nanocrystals: Successes and Challenges. ACS Energy Lett. 2019, 4, 709-719.

92. Cai, Y.; Wang, H.; Li, Y.; Wang, L.; Lv, Y.; Yang, X.; Xie, R.-J. Trimethylsilyl IodineMediated Synthesis of Highly Bright Red-Emitting $\mathrm{CsPbI}_{3}$ Perovskite Quantum Dots with Significantly Improved Stability. Chem. Mater. 2019, 31, 881-889.

93. Zhou, D.; Teng, H.; Koike, K.; Koike, Y.; Okamoto, Y. Copolymers of Methyl Methacrylate and Fluoroalkyl Methacrylates: Effects of Fluoroalkyl Groups on the Thermal and Optical Properties of the Copolymers. J. Polym. Sci. Pol. Chem. 2008, 46, 4748-4755.

94. Koike, K.; Kado, T.; Satoh, Z.; Okamoto, Y.; Koike, Y. Optical and Thermal Properties of Methyl Methacrylate and Pentafluorophenyl Methacrylate Copolymer: Design of Copolymers for Low-Loss Optical Fibers for Gigabit In-Home Communications. Polymer 2010, 51, 1377-1385.

95. Moquin, A.; Hanna, R.; Liang, T.; Erguven, H.; Gran, E. R.; Arndtsen, B. A.; Maysinger, D.; Kakkar, A. PEG-conjugated pyrrole-based polymers: one-pot multicomponent synthesis and self-assembly into soft nanoparticles for drug delivery. Chem. Commun. 2019, 55, 98299832.

96. Lin, K.; Wang, J.; Hu, Z.; Xu, R.; Liu, J.; Liu, X.; Xu, B.; Huang, F.; Cao, Y. Novel crosslinked films from epoxy-functionalized conjugated polymer and amine based small molecule for the interface engineering of high-efficiency inverted polymer solar cells. Sol. Energy Mater. Sol. Cells 2017, 168, 22-29.

\section{TOC Graphic}




Anchor Functional
group group

